\title{
Prospectiva e inteligencia competitiva al 2040 en los programas fomentan la innovación en el Perú
}

\section{RESUMEN}

El presente artículo, resume una investigación que tuvo como objetivo formular un escenario futurible al 2040 para los programas que fomentan la innovación en el Perú, en el marco de la inteligencia competitiva. Para el logro de dicho objetivo, se consideró una muestra de 10 expertos en el tema de diferentes organizaciones, y mediante la aplicación de entrevistas y encuestas se logró identificar 37 variables del sistema de las cuales 23 fueron consideradas como claves y con ellas se construyó el escenario futurible.

Al ser una investigación prospectiva y por ende basada en la consulta a expertos, la principal dificultad encontrada residió en la disponibilidad de estos; sin embargo esto fue superado.

Palabras clave: prospectiva, escenario, innovación, variable, sistema

Prospective And Competitive intelligence IN 2040 TO PROMOTE PROGRAMS INNOVATION IN Peru

\section{ABSTRACT}

This paper, resume a research that had as objetive build a future scenario to 2040 for programs that promote the innovation in Perú, inside the competitive intelligence. In order to achieve this goal, it considered a simple of 10 experts of differents organizations, and Through the implementation of interviews and surveys It was possible to identify 37 variables of the system, of which 23 were considered as keys, and with them built the future scenario.

It's a prospective research, therefore based on consultation with experts, the main difficulty encountered he resided in the availability of these; however, this was solved.

Keywords: prospective, scenario, innovation, variable, system

\section{INTRODUCCIÓN}

La prospectiva es la ciencia que estudia los hechos presentes, datos históricos entre otros con el fin de entender el comportamiento de las variables y poder construir escenarios futuribles. También es considerada como una "indisciplina" con rigor intelectual; es indisciplina porque se rebela ante la concepción de que el futuro es uno solo y además tiene un rigor intelectual porque se basa en una serie de métodos probados y validados [6].

Por otro lado, "La prospectiva no es una técnica, ni la aplicación de un conjunto de métodos o técnicas, eso sería LA FOTOGRAFÍA. La prospectiva es un procedimiento que toma en cuenta los factores del entorno dinámico y cambiante, además de la aplicación de técnicas, esto es LA PELíCULA" [1]; esta definición muestra mediante la comparación de que la prospectiva no es algo estático, dogmático o inamovible; todo lo contrario, la prospectiva se desarrolla en un entorno cambiante y sobre todo considera la incertidumbre.

Dos componentes importantes para la prospectiva son la anticipación y la acción; de hecho "La prospectiva estratégica pone la anticipación al servicio de la acción" [6]; es decir la prospectiva debe traducirse en acción; de los escenarios futuribles se desprenden las políticas que luego darán vida a los planes estratégicos, de tal forma que permitan lograr el escenario futurible, mediante la escalera prospectiva [2].

Como antecedente se puede mencionar la tesis doctoral Prospectiva estratégica aplicada a la universidad pública [3], en donde se realiza un estudio prospectivo a las universidades públicas tanto en pre como en posgrado cubriendo las variables claves detectadas; en dicha tesis para la selección de expertos se tomó una muestra no probabilística estratificada, considerando la división propuesta por la ANR.

Otro antecedente importante a considerar es el Estudio prospectivo para la enseñanza superior virtual al 2030 [8] en donde se publica los resultados del estudio, así como los

\footnotetext{
* Doctor en Ciencias Administrativas. Profesor del Departamento de Diseño y Tecnología Industrial. UNMSM. E-mail: jlinche@hotmail.com

** Doctor en Ingeniería Industrial. Profesor del Departamento de Diseño y Tecnología Industrial. UNMSM. E-mail: alfonso.chung@industrial.unmsm.pe

*** Magíster en Informática. Profesor del Departamento Académico de Ingeniería de Sistemas e Informática. UNMSM. E-mail: edgar.ruiz@industrial.unmsm.pe
} 
lineamientos generales del escenario futurible fruto de la aplicación de las técnicas de consulta a expertos.

Los resultados de la investigación son importantes puesto que permitirán orientas los diferentes planes estratégicos de las instituciones involucradas en el tema hacía un escenario futurible, es decir probable y posible, en donde los objetivos se orienten al logro de variables que tengan impacto significativo sobre el sistema y que a la vez sean realizables, es decir en donde los actores tengan el poder para cambiarlas; ya que muchas veces se proponen objetivos que no impactan significativamente sobre el estado actual del escenario o se plantean objetivos muy ambiciosos, que de hecho tiene un impacto notable pero que no son posibles de realizar. En ninguno de los dos casos mencionados habrá pues una mejora significativa en el estado del arte de las cosas.

La investigación logró responder a la pregunta problemática planteada y con ello a la hipótesis del estudio y al mismo tiempo de transforma en una poderosa herramienta de cambio, no solo por los resultados en sí, sino por el detalle del método que se presenta; ya que es una adaptación práctica de la caja de herramientas del Dr. Godet.

\section{METODOLOGÍA}

En cuanto a la metodología, la investigación fue aplicada con una tipología descriptiva, explicativa y prospectiva. La población fueron todos los expertos en los programas fomentan la innovación en el Perú, por lo tanto es infinita. La muestra estuvo conformada por 10 expertos cuyo muestreo fue no probabilístico y por conveniencia. A continuación, el Cuadro 1 presenta la lista de estos expertos.

Es preciso recordar que para la prospectiva, es más importante la calidad de los expertos que el tamaño o la aleatoriedad en su selección, toda vez que tiene un fuerte componente cualitativo que luego se buscará llevarlo a cuantitativo en la medida de los posible; por lo tanto la investigación también fue mixta (cualitativa y cuantitativa).

Por otro lado, para el levantamiento de datos, se utilizó la técnica de la entrevista con una grabadora como herramienta y la encuesta con un cuestionario también como herramienta [7]. Ambas herramientas fueron validadas por panel de expertos. Finalmente, para la construcción de escenarios, se utilizó el método Multi Escenarios.

\section{Identificación de las variables claves del sistema}

En la primera etapa se hizo las entrevistas a los expertos indicados en el Cuadro 1, y con ello se identificó 37 variables, las cuales se presentan a continuación (véase el Cuadro 2).

De estas 37 variables solo interesa aquellas que sean claves, es decir que puedan ser modificadas por los actores (dependientes) y que su cambio tenga un impacto significativo dentro del escenario en estudio (influyentes).

Cuadro 1. Muestra de expertos.

\begin{tabular}{|c|c|}
\hline NOMBRES Y APELLIDOS & INSTITUCIÓN \\
\hline Carlos Salazar García & Fondo para la Innovación, Ciencia y Tecnología (FINCyT) \\
\hline Patricia Ríos Enriquez & Fondo para la Innovación, Ciencia y Tecnología (FINCyT) \\
\hline Marco Álvarez & Fondo para la Innovación, Ciencia y Tecnología (FINCyT) \\
\hline Fernando Ortega San Martín & Consejo Nacional de Ciencia, Tecnología e Innovación (CONCYTEC) \\
\hline José Álvarez Merino & Consejo Nacional de Ciencia, Tecnología e Innovación (CONCYTEC) \\
\hline Patrick Villanueva Bartra & CIDE - Pontificia Universidad Católica del Perú \\
\hline Julio César Vela Velásquez & CIE - Universidad del Pacífico \\
\hline Karen Weinberger Villarán & Ministerio de relaciones exteriores \\
\hline Javier Lossio Olavarría & Beneficiario de WAYRA \\
\hline Domingo Seminario De Col &
\end{tabular}


Cuadro 2. Variables del sistema.

\begin{tabular}{|c|c|}
\hline $\mathbf{N} .^{\circ}$ & VARIABLE \\
\hline 1 & Inversión en tecnológica \\
\hline 2 & Generación de nuevos polos de tecnología \\
\hline 3 & $\begin{array}{l}\text { Generación de tecnología de acuerdo a las } \\
\text { necesidades de cada sector y región }\end{array}$ \\
\hline 4 & $\begin{array}{l}\text { Inversión económica en sectores tecnológicos no } \\
\text { clásicos que puedan hacer la diferenciación desde } \\
\text { Perú. }\end{array}$ \\
\hline 5 & $\begin{array}{l}\text { Fortalecimiento de capacidades profesionales } \\
\text { para dar soporte y continuidad a innovaciones } \\
\text { tecnológicas. }\end{array}$ \\
\hline 6 & $\begin{array}{l}\text { Articulación, integración y alineación de apoyo a } \\
\text { la innovación. }\end{array}$ \\
\hline 7 & Asociatividad de nuevos sectores \\
\hline 8 & Generación de clúster tecnológicos. \\
\hline 9 & Implementación de laboratorios \\
\hline 10 & Desarrollo de agendas sectoriales \\
\hline 11 & Uso ético de fondos designados a innovación. \\
\hline 12 & $\begin{array}{l}\text { Incorporación de investigadores o especialistas } \\
\text { en cada tema a las empresas. }\end{array}$ \\
\hline 13 & Calidad de productos o servicios. \\
\hline 14 & Mejora de procesos \\
\hline 15 & Índices de innovación \\
\hline 16 & Nivel de riesgo \\
\hline 17 & Rentabilidad \\
\hline 18 & Impacto ambiental \\
\hline 19 & Potencial de invención \\
\hline 20 & Potencial de creatividad \\
\hline 21 & Nivel de conocimiento \\
\hline 22 & Vigilancia tecnológica \\
\hline 23 & Influencia en el PBI \\
\hline 24 & Capacidad tecnológica \\
\hline 25 & Vinculación con la empresa \\
\hline 26 & $\begin{array}{l}\text { Desarrollo y continuidad de políticas de } \\
\text { promoción a la innovación }\end{array}$ \\
\hline 27 & Nivel de cooperación \\
\hline 28 & Desarrollo regional \\
\hline 29 & Integración fronteriza \\
\hline 30 & Desarrollo productivo \\
\hline 31 & $\begin{array}{l}\text { Generación de productos o servicios orientados } \\
\text { a la sociedad del conocimiento }\end{array}$ \\
\hline 32 & Capacidad de adaptación a los cambios \\
\hline 33 & Nivel de distribución de ingresos \\
\hline 34 & Nivel de cambio en la estructura de valores \\
\hline 35 & Proyectos en función a la biodiversidad \\
\hline 36 & $\begin{array}{l}\text { Disposición a compartir los beneficios de la } \\
\text { implementación de la tecnología }\end{array}$ \\
\hline 37 & Modelo educativo \\
\hline
\end{tabular}

\section{Identificación de las variables clave}

Para dicha identificación se aplicó dos cuestionarios, el primero para medir el nivel de influencia y el segundo para la dependencia, los resultados se presentan a continuación.

Cuadro 3. Determinación de las variables claves del sistema.

\begin{tabular}{|c|c|c|c|c|}
\hline N. ${ }^{\circ}$ & VARIABLE & 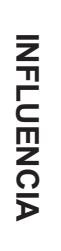 & 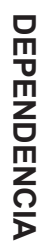 & 学离 \\
\hline 1 & Inversión en tecnológica & $\mathrm{x}$ & $\mathrm{x}$ & $x$ \\
\hline 2 & $\begin{array}{l}\text { Generación de nuevos polos } \\
\text { de tecnología }\end{array}$ & $x$ & & \\
\hline 3 & $\begin{array}{l}\text { Generación de tecnología de } \\
\text { acuerdo a las necesidades de } \\
\text { cada sector y región }\end{array}$ & $\mathrm{x}$ & $\mathrm{x}$ & $x$ \\
\hline 4 & $\begin{array}{l}\text { Inversión económica en } \\
\text { sectores tecnológicos no } \\
\text { clásicos que puedan hacer la } \\
\text { diferenciación desde Perú. }\end{array}$ & $x$ & & \\
\hline 5 & $\begin{array}{l}\text { Fortalecimiento de } \\
\text { capacidades profesionales } \\
\text { para dar soporte y continuidad } \\
\text { a innovaciones tecnológicas. }\end{array}$ & $x$ & $x$ & $\mathrm{x}$ \\
\hline 6 & $\begin{array}{l}\text { Articulación, integración y } \\
\text { alineación de apoyo a la } \\
\text { innovación. }\end{array}$ & $\mathrm{x}$ & $\mathrm{x}$ & $\mathrm{x}$ \\
\hline 7 & $\begin{array}{l}\text { Asociatividad de nuevos } \\
\text { sectores }\end{array}$ & $\mathrm{x}$ & $\mathrm{x}$ & $\mathrm{x}$ \\
\hline 8 & $\begin{array}{l}\text { Generación de clúster } \\
\text { tecnológicos. }\end{array}$ & $\mathrm{x}$ & & \\
\hline 9 & $\begin{array}{l}\text { Implementación de } \\
\text { laboratorios }\end{array}$ & $\mathrm{x}$ & $\mathrm{x}$ & $x$ \\
\hline 10 & $\begin{array}{l}\text { Desarrollo de agendas } \\
\text { sectoriales }\end{array}$ & & $\mathrm{x}$ & \\
\hline 11 & $\begin{array}{l}\text { Uso ético de fondos } \\
\text { designados a innovación. }\end{array}$ & & $x$ & \\
\hline 12 & $\begin{array}{l}\text { Incorporación de } \\
\text { investigadores o especialistas } \\
\text { en cada tema a las empresas. }\end{array}$ & $x$ & $x$ & $\mathrm{x}$ \\
\hline 13 & $\begin{array}{l}\text { Calidad de productos o } \\
\text { servicios. }\end{array}$ & $\mathrm{x}$ & $x$ & $x$ \\
\hline 14 & Mejora de procesos & & $\mathrm{x}$ & \\
\hline 15 & Índices de innovación & $x$ & $\mathrm{x}$ & $\mathrm{x}$ \\
\hline 16 & Nivel de riesgo & $x$ & $\mathrm{x}$ & $\mathrm{x}$ \\
\hline 17 & Rentabilidad & $\mathrm{x}$ & $\mathrm{x}$ & $\mathrm{x}$ \\
\hline
\end{tabular}




\begin{tabular}{|c|c|c|c|c|}
\hline 18 & Impacto ambiental & $x$ & $x$ & $x$ \\
\hline 19 & Potencial de invención & $x$ & $X$ & $x$ \\
\hline 20 & Potencial de creatividad & $x$ & $X$ & $x$ \\
\hline 21 & Nivel de conocimiento & $x$ & $x$ & $x$ \\
\hline 22 & Vigilancia tecnológica & $x$ & $x$ & $\mathrm{X}$ \\
\hline 23 & Influencia en el PBI & & $x$ & \\
\hline 24 & Capacidad tecnológica & $x$ & & \\
\hline 25 & Vinculación con la empresa & $x$ & $X$ & $x$ \\
\hline 26 & $\begin{array}{l}\text { Desarrollo y continuidad de } \\
\text { políticas de promoción a la } \\
\text { innovación }\end{array}$ & $x$ & & \\
\hline 27 & Nivel de cooperación & $x$ & $\mathrm{X}$ & $x$ \\
\hline 28 & Desarrollo regional & $x$ & $X$ & $x$ \\
\hline 29 & Integración fronteriza & $x$ & $X$ & $\mathrm{X}$ \\
\hline 30 & Desarrollo productivo & $x$ & $x$ & $x$ \\
\hline 31 & $\begin{array}{l}\text { Generación de productos } \\
\text { o servicios orientados a la } \\
\text { sociedad del conocimiento }\end{array}$ & $x$ & $X$ & $x$ \\
\hline 32 & $\begin{array}{l}\text { Capacidad de adaptación a } \\
\text { los cambios }\end{array}$ & $x$ & & \\
\hline 33 & $\begin{array}{l}\text { Nivel de distribución de } \\
\text { ingresos }\end{array}$ & & & \\
\hline 34 & $\begin{array}{l}\text { Nivel de cambio en la } \\
\text { estructura de valores }\end{array}$ & $x$ & & \\
\hline 35 & $\begin{array}{l}\text { Proyectos en función a la } \\
\text { biodiversidad }\end{array}$ & $x$ & $X$ & $x$ \\
\hline 36 & $\begin{array}{l}\text { Disposición a compartir } \\
\text { los beneficios de la } \\
\text { implementación de la } \\
\text { tecnología }\end{array}$ & $x$ & & \\
\hline 37 & Modelo educativo & $x$ & & \\
\hline
\end{tabular}

Como se puede apreciar hay 23 variables que son claves. A continuación se extraen del cuadro anterior y se presentan separadas para un mejor entendimiento del estudio.

- Inversión en tecnológica.

- Generación de tecnología de acuerdo a las necesidades de cada sector y región

- Fortalecimiento de capacidades profesionales para dar soporte y continuidad a innovaciones tecnológicas.

- Articulación, integración y alineación de apoyo a la innovación.

- Asociatividad de nuevos sectores

- Generación de clúster tecnológicos.
- Implementación de laboratorios

- Incorporación de investigadores o especialistas en cada tema a las empresas.

- Calidad de productos o servicios.

- Índices de innovación

- Nivel de riesgo

- Rentabilidad

- Impacto ambiental

- Potencial de invención

- Potencial de creatividad

- Nivel de conocimiento

- Vigilancia tecnológica

- Vinculación con la empresa

- Nivel de cooperación

- Desarrollo regional

- Integración fronteriza

- Desarrollo productivo

- Generación de productos o servicios orientados a la sociedad del conocimiento

- Proyectos en función a la biodiversidad

\section{Construcción del escenario futurible}

Para la construcción del escenario futurible es necesario generar un mini escenario por cada variable, la cual luego es validada por los expertos seleccionados para el estudio. Luego todos estos escenarios se unen en uno solo que luego fue validado nuevamente por los expertos, en ambos casos se utilizó el Delphi. Por lo tanto para el año 2040 el escenario futurible sería:

En el año 2040, los fondos que captan los programas que fomentan la innovación están en gran parte destinado a tecnología y son captados por los emprendedores, los cuales conocen la importancia de este factor.

Los participantes de los programas que fomentan la innovación han generado tecnología fruto de un estudio de las necesidades de su región y orientadas a la satisfacción de estas.

La generación de tecnología no es suficiente si es que no está acompaña del personal idóneo, es por eso que los programas que fomentan la innovación se han preocupado por la preparación de los emprendedores para el acceso a esta tecnología, es por eso que dichos participantes están debidamente capacitados y además 
son capaces de adaptarse a los cambios de paradigmas tecnológicos.

Los diferentes programas de apoyo a la innovación están articulados, integrados y alienadosm, según el escenario prospectivo propuesto; por lo tanto sus planes estratégicos y por ende sus actividades apuntan al logro de dicho escenario a nivel nacional.

Como resultado de la maduración de las innovaciones tecnológicas promovidas por los programas de fomento, han aparecido nuevos sectores los cuales se han agrupado en clústeres, los mismos que funcionan sin perder el contacto con las entidades que fomentaron los programas que originaron su formación.

Dentro de la asociatividad comentada anteriormente, los clústeres son fundamentalmente tecnológicos y han evolucionado bastante bien, tanto que están al nivel de lo que se conocía como Silicon Valley a inicios del siglo XXI.

La investigación experimental es muy importante para el desarrollo tecnológico, y así lo comprendieron las Universidades (tanto públicas como privadas); es así que desde el 2014 dichas universidades han ido incrementando su nivel de participación en los programas que fomentan la innovación, de tal manera que en la actualidad cuentan con laboratorios de tecnología de vanguardia, los cuales atienden las diversas necesidades en investigación.

La relación entre universidad y empresa ha sido debidamente potenciada, y como resultado de diversas capacitaciones (financiadas por programas que fomentan la innovación), las universidades tienen convenios con empresas mediante el cual diversos docentes investigadores, especialistas en determinados temas, hacen pasantías en las empresas resolviendo problemas específicos en su entorno.

La calidad es directamente proporcional a la satisfacción del cliente, si bien en el año 2014 esto no era aún entendido por los emprendedores; los programas de innovación fueron poco a poco creando una conciencia de calidad entre sus participantes, de tal manera que en la actualidad la calidad de los productos o servicios está acorde a los estándares y en muchos casos los supera.

La medición del nivel de innovación es un factor clave para poder determinar el nivel de avance y efectividad de los programas que fomentan la innovación; es por eso que en el 2015 las diversas organizaciones que fomentan estos programas se reunieron y acordaron un sistema estándar de medición de la innovación el mismo que en la actualidad ha dado mediciones que superan las metas propuestas.

El nivel de riesgo es otro factor importante y decisorio en cuanto a la acción de iniciar o no una innovación, por lo tanto los participantes de los programas en estudio conocen las técnicas para su cálculo, lo cual les ha facilitado hasta la fecha decisiones exitosas.

La rentabilidad es vital para la elección de una innovación tecnológica, si bien en el 2014 aún los emprendedores no tenían bien claro las técnicas para su cálculo, en la actualidad esto se ha revertido ya que generación tras generación han sido capacitados en este tema y en la actualidad lo manejan como parte de la evaluación de la innovación.

Con respecto al impacto ambiental, en la actualidad la tecnología y la conciencia en este tema ha avanzado tanto que ahora se prefieren innovaciones con impactos nulos o despreciables.

La cantidad de patentes (forma de medir las invenciones) ha aumentado en la actualidad en forma exponencial, si se consideran los índices que se tenían en el 2014 y esto se debe gracias al fomento de las innovaciones, y en la capacitación dirigida a los participantes sobre el tema.

Un factor principal que ha impulsado el aumento de patentes es el aumento del potencial creativo, y esto se manifiesta no solo en las patentes sino que también se materializa en la solución de problemas y también en la calidad de las innovaciones que se presentan a los concursos.

El nivel de conocimientos es también otra variable clave para el desarrollo sostenible de las innovaciones, y así también lo entendieron las organizaciones que fomentan los programas de innovación, de tal forma que desde el 2015 comenzaron a potenciar los convenios con universidades e institutos técnicos, de tal forma que los participantes han ido haciendo pasantías en dichas instituciones a fin de mejorar sus conocimientos y con ello lograron la sostenibilidad de sus innovaciones.

La vigilancia tecnológica, el cual era un concepto casi desconocido en el 2014, es ahora parte de todas las innovaciones que se proponen a los concursos; tanto así que ya es un conocimiento 
implantado en el desarrollo de las actividades de los innovadores.

Las instituciones que fomentan los programas de innovación han seguido políticas que los han integrado exitosamente a las empresas, de tal manera de que conocen su entorno y los diversos problemas que afrontan; esto también se ha visto potenciado por la participación de las universidades en dicha integración.

El nivel de cooperación entre las entidades que fomentan los programas de innovación y los emprendedores ha ido en constante aumento, y esto se traduce en el nivel cada vez más alto de participantes y en la calidad de sus propuestas. Asimismo también se ha visto favorecida la cooperación entre la universidad y estas instituciones, ya sea a través de la participación en el equipamiento de laboratorios, desarrollo de pasantías o reuniones de coordinación de actividades conjuntas.

El desarrollo regional también se ha visto favorecido a la fecha, ya que desde el 2014 se ha iniciado un proceso de descentralización y en la última medición se ha visto un incremento del desarrollo en las diversas áreas de las regiones.

A inicios del siglo XXI la integración fronteriza recién estaba en sus comienzos, sin embargo en la actualidad y gracias al desarrollo regional, las localidades fronterizas al logrado presionar al gobierno central para estar perfectamente articuladas en las políticas que de palacio de gobierno se emitan.

Uno de los factores claves para el desarrollo regional ha sido el desarrollo productivo, el cual vino como consecuencia de la aplicación de los programas de fomento de la innovación.

Debido al desarrollo tecnológico y al fomento en innovación, la mayoría de proyectos son tecnológicos (con valor agregado) y están orientados a la sociedad del conocimiento. Por lo tanto el peruano ha logrado entender que las necesidades del futuro van por ese sector y ahora con los conocimientos adquiridos, generación tras generación desde el 2014, se ha orientado a ese nuevo mercado.

Como parte de la comprensión del desarrollo sustentable, el cual se ha ido inculcando como parte de los programas de innovación; los proyectos en la actualidad (a diferencia de inicios del siglo $\mathrm{XXI}$ ) se articulan perfectamente con la naturaleza y respetan su normal desarrollo.

\section{Interpretación de datos}

El escenario futurible obtenido, si bien contiene algunas políticas que ya están en ejecución; también presenta otras variables que deben traducirse en políticas para lograr este futurible deseado. Asimismo debe potenciarse sobre todo al innovador y por ende la cultura de la innovación en el Perú.

Es importante recordar que "La innovación es una función crítica para las empresas. De su desarrollo estratégico se pueden construir ventajas competitivas saludables y sostenibles en el tiempo" [9]. Este concepto ha sido confirmado por el escenario futurible construido.

Por otrolado, cabe recordar que la economía peruana es pequeña y abierta [4] por lo tanto las variables mencionadas tendrán un impacto significativo, en caso que se trabajen adecuadamente sobre ellas.

"La ciencia, tecnología e innovación (CTI) resuelve problemas relacionados con la salud, obtención y utilización de energía, alimentos; mejor aprovechamiento de la tierra; suministro de agua potable, productividad, competitividad y crecimiento económico. Por ello, el desarrollo es imposible sin capacidades de innovación, ciencia y tecnología autónomas" [10]. Es por eso la importancia de la innovación en los procesos de integración fronteriza y de desarrollo regional, tal y como se ha planteado en el escenario futurible.

Sin embargo el Perú al año 2014 se encuentra en el puesto 73 en innovación, habiendo incluso retrocedido cuatro posiciones respecto al 2013 [5], por lo tanto el escenario propuesto adquiere una mayor importancia en cuanto a su puesta en marcha.

\section{CONCLUSIONES}

1. Debe fomentarse una cultura de innovación en el Perú.

2. Las universidades deben articularse tanto a las empresas como a los programas que fomentan la innovación en el país.

3. La formación de clústeres tecnológicos también deviene en una necesidad bajo el esquema del futurible planteado en la presenta investigación.

4. La formación de indicadores estándares para medir la innovación y que sean fruto de un consenso entre las entidades que fomentan estos programas, las universidades y el Estado es una necesidad prioritaria para medir el avance en este tema. 
5. Es prioritaria la formación de nuevos individuos con nuevas concepciones acera de la innovación y sobre todo su armonía con el medioambiente.

\section{4, REFERENCIAS BIBLIOGRÁFICAS}

[1] Baena, G. (2007). Técnicas de prospectiva social. REDIVU. En: http://www.redivu.org/ bvcprospectiva/9.pdf

[2] Chung A. (2009). Prospectiva estratégica: Más allá del Plan Estratégico. Industrial Data, 12(2): pp 27-31

[3] Chung A. (2012). Prospectiva estratégica aplicada a la universidad pública. Tesis para optar el grado de doctor en ingeniería industrial. UNMSM

[4] Diaz J. y Kuramoto J. (2010). Evaluación de políticas de apoyo a la innovación en el Perú. Ministerio de Economía y Finanzas. Gobierno del Perú.

[5] El Economista (2014). CCL: Avance de innovación en el Perú dependerá de un marco institucional sólido. En: http://www. eleconomistaamerica.pe/economia-eAmperu/noticias/6154520/10/14/CCL-Avancede-innovacion-en-el-Peru-dependera-de-unmarco-institucional-solido.html

[6] Godet M. ,2009, Caja de Herramientas de la Prospectiva Estratégica, Edición Digital, LIPSOR: Francia

[7] Hernández R.; Fernández, C. y Baptista, P (2010). Metodología de la Investigación. McGraw-Hill: México

[8] Inche J. y Chung A. (2012). Estudio prospectivo para la enseñanza superior virtual al 2030 Industrial Data, 15(1), 120-126.

[9] Mayorga D. y García E. (2010). Innovación empresarial en el Perú. Incae Business Review, 10(1), 1-7.

[10] Ministerio de Relaciones Exteriores (2012). Ciencia Tecnología e Innovación. En: http:// www.rree.gob.pe/temas/Paginas/Ciencia_ Tecnologia_e_Innovacion.aspx 